MATHEMATICS OF COMPUTATION

Volume 65, Number 216

October 1996, Pages 1567-1581

\title{
ON WEIGHT FUNCTIONS WHICH ADMIT EXPLICIT GAUSS-TURÁN QUADRATURE FORMULAS
}

\author{
LAURA GORI AND CHARLES A. MICCHELLI
}

\begin{abstract}
The main purpose of this paper is the construction of explicit Gauss-Turán quadrature formulas: they are relative to some classes of weight functions, which have the peculiarity that the corresponding s-orthogonal polynomials, of the same degree, are independent of $s$. These weights too are introduced and discussed here. Moreover, highest-precision quadratures for evaluating Fourier-Chebyshev coefficients are given.
\end{abstract}

\section{INTRODUCTION}

Given a function $w$ which is positive and integrable on the interval $[-1,1]$, the ze$\operatorname{ros} x_{1}, \ldots, x_{n}$ of the $n$ th-degree orthogonal polynomial corresponding to $w$ provide the nodes of a quadrature rule for the integral

$$
I(f ; w):=\int_{-1}^{1} f(x) w(x) d x
$$

which is of maximum degree of precision. That is, there are positive weights $\lambda_{1}, \lambda_{2}, \ldots, \lambda_{n}$ such that

$$
I(f ; w)=Q_{0}(f ; w):=\sum_{j=1}^{n} \lambda_{j} f\left(x_{j}\right), \quad f \in \pi_{2 n-1},
$$

where $\pi_{k}:=$ the space of all polynomials of degree $\leq k$. Moreover, there is no formula using a linear combination of $n$ values of $f$ that gives $I(f ; w)$ for all polynomials of degree $2 n$. This classical result on "Gaussian quadrature" was extended by Turán in his interesting paper [13]. Turán considered quadrature rules of the form

$$
Q_{s}(f ; w):=\sum_{k=0}^{2 s} \sum_{j=1}^{n} \lambda_{k j} f^{(k)}\left(x_{j, s}\right)
$$

and showed that such rules have a maximum degree of precision $2(s+1) n-1$. Moreover, he showed that the $n$ zeros $x_{1, s}, \ldots, x_{n, s}$ of the monic polynomial of

Received by the editor November 29, 1994 and, in revised form, August 9, 1995.

1991 Mathematics Subject Classification. Primary 65D32; Secondary 41A55.

Key words and phrases. Quadrature, Turán-type integration rules, generalized Jacobi weights.

The second author was partially supported by the Alexander von Humboldt Foundation.

The first author was supported by Ministero Università e Ricerca Scientifica e Tecnologica Italia.

(C)1996 American Mathematical Society 
degree $n$ which minimizes the expression

$$
\int_{-1}^{1}|p(x)|^{2 s+2} w(x) d x
$$

over all such polynomials gives a quadrature rule of maximum degree of accuracy,

$$
I(f ; w)=Q_{s}(f ; w), \quad f \in \pi_{2(s+1) n-1} .
$$

Turán's elegant extension of Gauss quadrature attracted considerable interest and still remains an attractive area of investigation. For instance, Gauss-Turán formulas are dealt with in the book [2], and the numerical problem of computing Turán formulas was studied in [3] and also [11], while an application to singular integrals is treated in [5].

Even in the case $s=1$, Turán left unsettled in [13] the determination of the signs of $\lambda_{0 j}$ and $\lambda_{1 j}$ in his formula. Using some facts about monosplines, one of us proved in [8] that alternate weights are always positive, namely,

$$
\lambda_{k j}>0, k=0,2, \ldots, 2 s, j=1,2, \ldots, n .
$$

Later, it was shown in [9] for the Chebyshev weight

$$
w_{\infty}(x):=\left(1-x^{2}\right)^{-1 / 2}, \quad x \in(-1,1),
$$

that $\lambda_{k, j}, j=1, \ldots, n$, can be both positive and negative. Explicit formulas for all the Gauss-Turán formulas corresponding to this weight function were also given in [9], in terms of certain divided difference functionals at the zeros of the $n$th Chebyshev polynomial,

$$
\begin{gathered}
T_{n}(x)=\cos n \theta, x=\cos \theta, \theta \in[0, \pi], \\
\xi_{j}=\cos [(2 j-1) \pi / 2 n], \quad j=1,2, \ldots, n .
\end{gathered}
$$

In another paper [10], these ideas were extended and also related to the work in [7] and [2] on certain periodic versions of the Gauss-Turán formulas. This additional information allowed the identification of the asymptotic behavior of $\lambda_{k j}$ corresponding to the Chebyshev weight function $w_{\infty}$ when $n \rightarrow \infty$ and $s$ is fixed, a problem raised in [14].

Recently, it was observed by one of us in [4] that for the class of weight functions

$$
w_{2, \mu}(x):=|x|^{2 \mu+1}\left(1-x^{2}\right)^{\mu}, \quad \mu>-1,
$$

explicit Gauss-Turán quadrature formulas can be given for all $s$, at least when $n=2$. Convergence properties of these formulas as $s \rightarrow \infty$ were studied in [4], and later in [6] these new quadrature formulas were used for the efficient computation of Cauchy principal value integrals.

The weight functions (1.10) studied in [4] and [6] fall into the category of "generalized Jacobi weights", which have been studied from other points of view in [1], [12], among others.

In this paper, sparked by the observations made in [4] about Gauss-Turán quadrature formulas, we introduce for each $n$ a class of weight functions (which include certain generalized Jacobi weight functions) for which explicit Gauss-Turán quadrature formulas of all orders can be found. Our results therefore extend and unify some of the results in [4] and [9].

The paper is organized as follows. In $\S 2$, we define the class of weight functions which are of interest to us. We then develop some of their properties and also 
give a few examples. Section 3 contains extensions and improvements on results from [9] and [10]. Finally, the last section contains explicit Gauss-Turán quadrature formulas for the class of weight functions described in $\S 2$.

\section{Weight FUnCtions}

The Fourier-Chebyshev expansion of a function $f$ defined and integrable on $[-1,1]$ is given by

$$
\sum_{n=0}^{\infty} A_{n} T_{n}(x)
$$

where

$$
A_{n}=A_{n}(f)=\frac{2}{\pi} \int_{-1}^{1} f(x) T_{n}(x) w_{\infty}(x) d x
$$

are its corresponding Fourier-Chebyshev coefficients. The prime on the summation indicates that the term corresponding to $n=0$ is halved.

For each $n$, we define the class $\mathcal{W}_{n}$ to consist of all nonnegative integrable functions $w$ on $[-1,1]$ such that the function $w / w_{\infty}$ has a Fourier-Chebyshev series of the form

$$
w / w_{\infty}=\sum_{\ell=0}^{\infty} \rho_{\ell} T_{2 \ell n},
$$

where convergence holds relative to the weighted $L^{1}$-norm

$$
\int_{-1}^{1}|f(x)| w_{\infty}(x) d x
$$

Accordingly, for every $w \in \mathcal{W}_{n}$ and $f \in C[-1,1]$ we have

$$
I(f ; w)=\frac{\pi}{2} \sum_{\ell=0}^{\infty} \rho_{\ell} A_{2 \ell n}(f) .
$$

In particular, it follows that

$$
I(f ; w)=\frac{\rho_{0}}{2} \int_{-1}^{1} f(x) w_{\infty}(x) d x, \quad f \in \pi_{2 n-1} .
$$

Consequently, if $p_{k}, k=0,1, \ldots$, are the polynomials orthogonal relative to $w$, normalized so that $p_{0}(x)=1$, and

$$
p_{k}(x)=2^{k-1} x^{k}+\cdots, \quad k \geq 1,
$$

then (2.6) implies that

$$
p_{k}=T_{k}, \quad k=0,1, \ldots, n .
$$

Moreover, recalling the fact that

$$
\int_{-1}^{1} f(x) w_{\infty}(x) d x=\frac{\pi}{n} \sum_{j=1}^{n} f\left(\xi_{j}\right), \quad f \in \pi_{2 n-1}
$$


(cf. [2]), we conclude that the Gauss quadrature formula for any $w \in \mathcal{W}_{n}$ is likewise given by

$$
I(f ; w)=\frac{\pi \rho_{0}}{2 n} \sum_{j=1}^{n} f\left(\xi_{j}\right), \quad f \in \pi_{2 n-1} .
$$

This formula is our first indication that it is feasible that explicit Gauss-Turán quadrature formulas for any $w \in \mathcal{W}_{n}$ can be found. In fact, (2.9) accomplishes this goal for $s=0$, the Gaussian case.

The first step in our quest for Gauss-Turán quadrature formulas identifies the $s$-orthogonal polynomials of degree $n$ for any $w \in \mathcal{W}_{n}$.

Proposition 2.1. Let $w \in \mathcal{W}_{n}$ and $1 \leq \gamma<\infty$. Then

$$
\min \left\{\int_{-1}^{1}\left|T_{n}(x)-p(x)\right|^{\gamma} w(x) d x: p \in \pi_{n-1}\right\}=\int_{-1}^{1}\left|T_{n}(x)\right|^{\gamma} w(x) d x .
$$

Specializing this result to $\gamma=2 s+2$ implies that the $n$-th degree $s$-orthogonal polynomial relative to the weight function $w$ is $T_{n}$ (independently of $s$ ).

Proof. For every polynomial $p$ we have

$$
\int_{-1}^{1}\left|T_{n}(x)-p(x)\right|^{\gamma} w(x) d x=\int_{0}^{\pi}|\cos n \theta-p(\cos \theta)|^{\gamma} g(\theta) d \theta
$$

where

$$
g(\theta):=w(\cos \theta)|\sin \theta|, \quad \theta \in[-\pi, \pi] .
$$

According to (2.3),

$$
g(\theta)=\sum_{\ell=0}^{\infty} \rho_{\ell} \cos 2 \ell n \theta, \quad \text { a.e. } \theta \in[-\pi, \pi],
$$

and hence

$$
g(\theta)=g\left(\theta+\frac{\pi}{n}\right), \quad \text { a.e. } \theta \in[-\pi, \pi]
$$

Now, without loss of generality we suppose that $1<\gamma<\infty$, and therefore the polynomial $p^{0} \in \pi_{n-1}$ which minimizes the left-hand side of (2.10) is unique. Using equation (2.13) and also the fact that

$$
\cos n\left(\theta+\frac{\pi}{n}\right)=-\cos n \theta
$$

we conclude that the function

$$
q^{0}(\theta):=p^{0}(\cos \theta)
$$

necessarily satisfies the equation

$$
q^{0}\left(\theta+\frac{\pi}{n}\right)=-q^{0}(\theta)
$$

Next, we express $q^{0}$ in the form

$$
q^{0}(\theta)=\sum_{|j| \leq n-1} q_{j} e^{i j \theta}
$$

for some constants $q_{j},|j| \leq n-1$. Then formula (2.15) implies that

$$
q_{j}\left(1+e^{i j \frac{\pi}{n}}\right)=0, \quad|j| \leq n-1,
$$

from which we conclude that $q^{0}=0$. 
We end this section with an example of a family of weight functions in $\mathcal{W}_{n}$. Recall that the $(n-1)$ st-degree Chebyshev polynomial $U_{n-1}$ of the second kind is given by

$$
U_{n-1}(\cos \theta)=\frac{\sin n \theta}{\sin \theta}, \quad \theta \in[0, \pi] .
$$

For every $\mu>-1$ we consider the generalized Gegenbauer weight

$$
w_{n, \mu}(x):=\left|U_{n-1}(x) / n\right|^{2 \mu+1}\left(1-x^{2}\right)^{\mu}, \quad x \in[-1,1] .
$$

When $n=2$ we get

$$
w_{2, \mu}(x)=|x|^{2 \mu+1}\left(1-x^{2}\right)^{\mu}, \quad x \in[-1,1],
$$

which is the weight function studied in [4] and [6]. In general, we have

$$
w_{n, \mu}(\cos \theta)|\sin \theta|=n^{-2 \mu-1}|\sin n \theta|^{2 \mu+1},
$$

and so for all $n=1,2, \ldots$ and $\mu>-1$

$$
w_{n, \mu} \in \mathcal{W}_{n}
$$

Moreover, we have

$$
\int_{-1}^{1} T_{2 \ell n}(x) w_{n, \mu}(x) d x=\kappa_{\ell} / n^{2 \mu+1}, \quad \ell=0,1, \ldots,
$$

where

$$
\kappa_{\ell}:=\int_{-1}^{1} T_{2 \ell}(x)\left(1-x^{2}\right)^{\mu} d x, \quad \ell=0,1, \ldots .
$$

Thus, we obtain for $f \in C[-1,1]$

$$
I\left(f ; w_{n, \mu}\right)=\frac{1}{n^{2 \mu+1}} \sum_{\ell=0}^{\infty} \kappa_{\ell} A_{2 \ell n}(f),
$$

where

$$
I\left(f ; w_{n, \mu}\right)=\int_{-1}^{1} f(x) w_{n, \mu}(x) d x .
$$

\section{Divided Difference functionals at the Chebyshev nodes}

To obtain explicit expressions for the Gauss-Turán quadrature formulas for weight functions in $\mathcal{W}_{n}$ we need to review some results from [9] and, at the same time, provide improvements and extensions of them.

We begin by recalling the form of the generating function of the Chebyshev polynomials. Specifically, for $x \in[-1,1]$ and complex $t$ in the unit disc, viz. $|t|<1$, we have

$$
\frac{1-t^{2}}{1-2 x t+t^{2}}=2 \sum_{j=0}^{\infty} t^{j} T_{j}(x) .
$$

We write the left-hand side of equation (3.1) in the form

$$
G_{t}(x):=\frac{\alpha(t)}{x-\beta(t)},
$$

where

$$
\alpha(t):=\left(t-t^{-1}\right) / 2
$$


and

$$
\beta(t):=\left(t+t^{-1}\right) / 2 .
$$

Therefore, (3.1) takes the form

$$
G_{t}=2 \sum_{j=0}^{\infty} t^{j} T_{j}
$$

Observe that to express a linear functional $L(f)$ in a series of Fourier-Chebyshev coefficients is tantamount to identifying the constants $L\left(T_{k}\right), k=0,1, \ldots$, which can be found directly from (3.5) by expanding $L\left(G_{t}\right)$ in a power series in $t$. We consider the functionals

$$
\mathcal{L}_{0}(f):=\sum_{j=1}^{n} f\left(\xi_{j}\right)
$$

and

$$
\mathcal{L}_{k}(f):=f^{\prime}\left(\xi_{1}^{k}, \ldots, \xi_{n}^{k}\right), \quad k=1,2, \ldots,
$$

where the last functional signifies the divided difference of $f^{\prime}$ at the points $\xi_{1}, \ldots, \xi_{n}$ each repeated with multiplicity $k$. Lemmas 1 and 2 in [9] provide expansions of these functionals in terms of the Fourier-Chebyshev coefficients of $f$. To explain these results, we introduce the functions

$$
g_{k}(z):=z^{k}\left(1-z^{2}\right) /\left(1+z^{2}\right)^{k+1}, \quad k=0,1, \ldots,
$$

each of which has a power series expansion in the unit disc

$$
g_{k}(z)=\sum_{\ell=0}^{\infty} g_{k \ell} z^{\ell}, \quad|z|<1
$$

whose coefficients are given by

$$
g_{0 \ell}=\left\{\begin{array}{ll}
1, & \ell=0, \\
2(-1)^{j}, & \ell=2 j, \quad j \geq 1, \\
0, & \text { otherwise },
\end{array} \quad g_{1 \ell}= \begin{cases}(-1)^{j}, & \ell=2 j+1, \\
0, & \text { otherwise }\end{cases}\right.
$$

and for $k \geq 2$

$$
g_{k \ell}= \begin{cases}(-1)^{j} \frac{(j+1) \cdots(j+k-1)(2 j+k)}{k !}, & \ell=2 j+k, \\ 0, & \text { otherwise. }\end{cases}
$$

Lemma 3.1. For every $t$ in the unit disc,

$$
\mathcal{L}_{0}\left(G_{t}\right)=n g_{0}\left(t^{n}\right)
$$

and for $k \geq 1$

$$
\mathcal{L}_{k}\left(G_{t}\right)=n k 2^{n k} g_{k}\left(t^{n}\right) .
$$

Proof. First we prove (3.12). To this end, we observe that

$$
\begin{aligned}
\mathcal{L}_{0}\left(G_{t}\right) & =\alpha(t) \sum_{j=1}^{n} \frac{1}{\xi_{j}-\beta(t)} \\
& =-\alpha(t) T_{n}^{\prime}(\beta(t)) / T_{n}(\beta(t)) .
\end{aligned}
$$


Since

$$
T_{n}(\beta(t))=\left(t^{n}+t^{-n}\right) / 2, \quad t \in \mathbb{C} \backslash\{0\},
$$

it follows that

$$
T_{n}^{\prime}(\beta(t))=n\left(t^{n}-t^{-n}\right) /\left(t-t^{-1}\right), \quad t \in \mathbb{C} \backslash\{0\},
$$

and so substituting these formulas in (3.14) gives

$$
\begin{aligned}
\mathcal{L}_{0}\left(G_{t}\right) & =n\left(t^{-1}-t\right)\left(t^{n}-t^{-n}\right) /\left(t-t^{-1}\right)\left(t^{n}+t^{-n}\right) \\
& =n\left(1-t^{2 n}\right) /\left(1+t^{2 n}\right)=n g_{0}\left(t^{n}\right) .
\end{aligned}
$$

For the proof of (3.13) we use the easily verified fact that for any $x_{1}, \ldots, x_{m}$ and $z \in \mathbb{C} \backslash\left\{x_{1}, \ldots, x_{m}\right\}$

$$
h_{z}\left(x_{1}, \ldots, x_{m}\right)=\frac{1}{\left(z-x_{1}\right) \cdots\left(z-x_{m}\right)},
$$

where

$$
h_{z}(x):=\frac{1}{z-x} .
$$

Equation (3.17) even holds if the points $x_{1}, \ldots, x_{m}$ are not distinct. In particular, by specializing (3.17) to the nodes

$$
\left\{x_{k 1}, \ldots, x_{k n}\right\}:=\{\underbrace{\xi_{1}, \ldots, \xi_{1}}_{k}, \ldots, \underbrace{\xi_{n}, \ldots, \xi_{n}}_{k}\},
$$

differentiating both sides of (3.17) with respect to $z$, we get

$$
h_{z}^{\prime}\left(\xi_{1}^{k}, \ldots, \xi_{n}^{k}\right)=k 2^{(n-1) k} T_{n}^{\prime}(z) / T_{n}^{k+1}(z),
$$

and consequently

$$
\mathcal{L}_{k}\left(G_{t}\right)=-k \alpha(t) 2^{(n-1) k} T_{n}^{\prime}(\beta(t)) / T_{n}^{k+1}(\beta(t)) .
$$

Once again, we appeal to equations (3.14) and (3.15) and after some simplification (3.13) follows.

This result leads to a series expansion of $\mathcal{L}_{k} f$ in terms of the Fourier-Chebyshev coefficients of $f$. In particular, for $f=G_{t}$ where $|t|<1$ we have from (3.5) that

$$
A_{j}\left(G_{t}\right)=2 t^{j}, \quad j=0,1, \ldots,
$$

and so for $k \geq 1$

$$
\mathcal{L}_{k}\left(G_{t}\right)=n k 2^{n k-1} \sum_{j=0}^{\infty} g_{k, 2 j+k} A_{(2 j+k) n}\left(G_{t}\right) .
$$

Therefore, for any $f \in \mathcal{G}:=$ algebraic $\operatorname{span}\left\{G_{t}:|t|<1\right\}$ we conclude that

$$
\mathcal{L}_{k}(f)=n k 2^{n k-1} \sum_{j=0}^{\infty} g_{k, 2 j+k} A_{(2 j+k) n}(f) .
$$

Similarly, for the same functions $f \in \mathcal{G}$

$$
\mathcal{L}_{0}(f)=\frac{n}{2} \sum_{j=0}^{\infty} g_{0,2 j} A_{2 j n}(f) .
$$

Our goal is to invert these formulas, that is, to solve for the Fourier-Chebyshev coefficients of $f$ in terms of the linear functionals $\mathcal{L}_{k}(f), k=0,1, \ldots$; of course, 
only multiples of $n$ can be found from the functionals $\mathcal{L}_{k}$. We do this in two stages. First, for each $\ell \geq 0$ we solve for $A_{2 \ell n}(f)$ as a linear combination of $\mathcal{L}_{2 k}(f), k=0,1, \ldots$. Then for each $\ell \geq 0$, we solve for $A_{(2 \ell+1) n}(f)$ as a linear combination of $\mathcal{L}_{2 k+1}(f), k=1,2, \ldots$ In each case it is helpful to express equations (3.23) and (3.24) in matrix notation.

For the first case, we introduce the upper triangular matrix $G=\left(G_{k \ell}\right)_{k, \ell=0,1, \ldots}$ whose elements are defined as

$$
G_{k \ell}= \begin{cases}\frac{n}{2} g_{0,2 \ell}, & k=0 \\ n k 4^{n k} g_{2 k, 2 \ell}, & \ell \geq k \geq 1 \\ 0, & \ell<k\end{cases}
$$

Then, replacing $k$ by $2 k$ in (3.23), we get

$$
\left(\mathcal{L}_{0}(f), \mathcal{L}_{2}(f), \ldots\right)^{T}=G\left(A_{0}(f), A_{2 n}(f), \ldots\right)^{T} .
$$

Since the elements of $G$ on its main diagonal are nonzero, $G$ has a unique upper triangular inverse. This matrix will allow us to invert equation (3.26), and therefore we identify it in the next lemma.

Lemma 3.2. Let $H=\left(H_{k \ell}\right)_{k, \ell=0,1, \ldots}$ be the upper triangular matrix defined for $k, \ell \geq 1$ by

$$
\sum_{\ell=1}^{\infty} H_{k \ell} \ell z^{\ell}=n^{-1} 4^{(n-1) k} z^{-k}\left(1-\sqrt{1-4^{-n+1} z}\right)^{2 k}\left(1-4^{-n+1} z\right)^{-1 / 2},|z|<4^{n-1},
$$

for $k=0, \ell \geq 1$ by

$$
\sum_{\ell=1}^{\infty} H_{0 \ell} \ell z^{\ell}=n^{-1}\left(\left(1-4^{-n+1} z\right)^{-1 / 2}-1\right), \quad|z|<4^{n-1},
$$

and

$$
H_{k 0}= \begin{cases}\frac{2}{n}, & k=0 \\ 0, & k \geq 1\end{cases}
$$

Then

$$
H=G^{-1} .
$$

Proof. According to the definition of $H$ we have for all $\tau$ in the unit disc and $k \geq 0$ that

$$
\frac{n}{2} H_{k 0} \sqrt{1-\tau}+\sum_{r=1}^{\infty} H_{k r} n r 4^{(n-1) r} \sqrt{1-\tau} \tau^{r}=\left(\frac{1-\sqrt{1-\tau}}{1+\sqrt{1-\tau}}\right)^{k} .
$$

Now, choose any $t$ in $(-1,1)$ and observe that $\left|T_{n}(\beta(t))\right|>1$ and set $\tau:=T_{n}^{-2}(\beta(t))$ in (3.30). Recalling that

$$
T_{n}(\beta(t))=\left(t^{n}+t^{-n}\right) / 2
$$

we see that

$$
1-\tau=\left(\frac{1-t^{2 n}}{1+t^{2 n}}\right)^{2}
$$


and therefore

$$
t^{2 n}=\frac{1-\sqrt{1-\tau}}{1+\sqrt{1-\tau}}
$$

Substituting these equations into (3.30) gives

$$
\frac{n}{2} H_{k 0} \frac{1-t^{2 n}}{1+t^{2 n}}+\sum_{r=1}^{\infty} H_{k r} n r 4^{n r} t^{2 r n} \frac{1-t^{2 n}}{\left(1+t^{2 n}\right)^{2 r+1}}=t^{2 k n}
$$

or equivalently

$$
\frac{n}{2} H_{k 0} g_{0}\left(t^{n}\right)+\sum_{r=1}^{\infty} H_{k r} n r 4^{n r} g_{2 r}\left(t^{n}\right)=t^{2 k n} .
$$

Moreover, from (3.25) we see that

$$
\sum_{\ell=0}^{\infty} G_{r \ell} t^{2 \ell n}= \begin{cases}\frac{n}{2} g_{0}\left(t^{n}\right), & r=0 \\ n r 4^{n r} g_{2 r}\left(t^{n}\right), & r \geq 1 .\end{cases}
$$

Therefore, we get

$$
\sum_{\ell=0}^{\infty} \sum_{r=0}^{\infty} H_{k r} G_{r \ell} t^{2 \ell n}=t^{2 k n}
$$

which proves

$$
\sum_{r=0}^{\infty} H_{k r} G_{r \ell}=\delta_{k \ell}, \quad k, \ell=0,1, \ldots
$$

To present the next result, we let $\Gamma_{n}$ denote the lemniscate $\left\{z:\left|T_{n}(z)\right|=1\right\}$. The function $z=\beta(t)$ gives a 1-to- 1 conformal mapping of the unit disc $|t|<1$ onto the extended complex plane with the segment $[-1,1]$ deleted. Hence the preimage of the exterior of the lemniscate under this map is the domain

$$
D_{n}:=\left\{t:|t|<1,\left|T_{n}(\beta(t))\right|>1\right\} .
$$

This is a symmetric subset of the unit disc which includes the interval $(-1,1)$. Let $R$ be a region which contains the lemniscate $\Gamma_{n}$ in its interior and $A(R)$ the class of all functions holomorphic in $R$.

Theorem 3.1. Let $f \in A(R)$. Then for all $k \geq 0$

$$
A_{2 k n}(f)=\sum_{r=0}^{\infty} H_{k r} \mathcal{L}_{2 r}(f) .
$$

In the case $k=0$ equation (3.28) implies that

$$
H_{0 r}=\frac{(-1)^{r}}{n r}\left(\begin{array}{c}
-\frac{1}{2} \\
r
\end{array}\right) 4^{-(n-1) r}, \quad r=1,2, \ldots
$$

and (3.29) gives

$$
H_{00}=\frac{2}{n} .
$$

Thus, setting

$$
\alpha_{j}:=\frac{(-1)^{j}}{2 j 4^{(n-1) j}}\left(\begin{array}{c}
-\frac{1}{2} \\
j
\end{array}\right), \quad j=1,2, \ldots
$$


we get from Theorem 3.1 the formula

$$
\begin{aligned}
\int_{-1}^{1} f(x) \frac{d x}{\sqrt{1-x^{2}}} & =\frac{\pi}{2} \sum_{r=0}^{\infty} H_{0 r} \mathcal{L}_{2 r}(f) \\
& =\frac{\pi}{n}\left\{\mathcal{L}_{0}(f)+\sum_{j=1}^{\infty} \alpha_{j} \mathcal{L}_{2 j}(f)\right\} .
\end{aligned}
$$

Formula (3.41) was proved in [9], where it was pointed out that the partial sums of the series in (3.41) provide the Gauss-Turán formula for the Chebyshev weight. Specifically, specializing (3.41) yields

$$
\int_{-1}^{1} f(x) \frac{d x}{\sqrt{1-x^{2}}}=\frac{\pi}{n}\left\{\sum_{j=1}^{n} f\left(\xi_{j}\right)+\sum_{j=1}^{s} \alpha_{j} f^{\prime}\left(\xi_{1}^{2 j}, \ldots, \xi_{n}^{2 j}\right)\right\}
$$

for all $f \in \pi_{2(s+1) n-1}$. Moreover, the right-hand side of (3.42) has the Gauss-Turán form (1.3).

The case $k \geq 1$ of Theorem 3.1 likewise yields Gauss-Turán formulas for the Fourier-Chebyshev coefficients of $f$. Namely, for any $s \geq 1$ we have

$$
A_{2 k n}(f)=\sum_{j=1}^{s} H_{k j} f^{\prime}\left(\xi_{1}^{2 j}, \ldots, \xi_{n}^{2 j}\right), \quad f \in \pi_{2(s+1) n-1} .
$$

Proof. The main idea of the proof is covered in the proof of Lemma 3.2. First, we point out that for $t \in D_{n}$ and $f=G_{t}$ equation (3.37) reduces to (3.35) by using Lemma 3.1. Hence, (3.37) has been proved for this case .

Now, let $f \in A(R)$ and choose a $\delta>0$ so that the simple closed curve

$$
\Gamma:=\left\{z:\left|T_{n}(z)\right|=1+\delta\right\}
$$

is contained in $R$ and contains $\Gamma_{n}$ in its interior. For $x \in[-1,1]$, the Cauchy integral formula gives

$$
f(x)=\frac{1}{2 \pi i} \int_{\Gamma} \frac{f(\zeta)}{\zeta-x} d \zeta .
$$

Every $\zeta \in \Gamma$ corresponds to a $t \in D_{n}$ with $\zeta=\beta(t)$. Therefore,

$$
\begin{aligned}
\mid A_{2 k n}(f) & -\sum_{\ell=1}^{m} H_{k \ell} \mathcal{L}_{2 k}(f) \mid \\
& \leq \frac{1}{2 \pi} \int_{\Gamma}|\alpha(t)|^{-1}|f(\zeta)|\left|A_{2 k}\left(G_{t}\right)-\sum_{\ell=1}^{m} H_{k \ell} \mathcal{L}_{2 \ell}\left(G_{t}\right)\right||d \zeta| .
\end{aligned}
$$

According to (3.35) the integrand goes to zero as $m \rightarrow \infty$, thereby establishing the result.

Next, we turn our attention to the second case mentioned earlier. Namely, we shall now find a formula for the Fourier-Chebyshev coefficients $A_{(2 k+1) n}(f), k \geq 0$, in terms of the functionals $\mathcal{L}_{2 \ell+1}(f), \ell \geq 0$. For this purpose, we introduce another upper triangular matrix $\hat{G}=\left(\hat{G}_{k \ell}\right)_{k, \ell=0,1, \ldots}$ defined by

$$
\hat{G}_{k \ell}= \begin{cases}n(2 k+1) 2^{n(2 k+1)-1} g_{2 k+1,2 \ell+1}, & \ell \geq k, \\ 0, & 0 \leq \ell<k .\end{cases}
$$


Then (3.23) implies that for $f \in \mathcal{G}$

$$
\left(\mathcal{L}_{1}(f), \mathcal{L}_{3}(f), \ldots\right)^{T}=\hat{G}\left(A_{n}(f) A_{3 n}(f), \ldots\right)^{T},
$$

and so again we are faced with the problem of finding the upper triangular inverse of a prescribed upper triangular matrix. In this case, the matrix is $\hat{G}$. To facilitate the identification of $\hat{G}^{-1}$, we observe from (3.9) and (3.44) that

$$
\sum_{\ell=0}^{\infty} \hat{G}_{k \ell} t^{(2 \ell+1) n}=n(k+1 / 2) 2^{n(2 k+1)} g_{2 k+1}\left(t^{n}\right), \quad|t|<1 \in D_{n} .
$$

The next lemma follows from this formula.

Lemma 3.3. Define the upper triangular matrix $\hat{H}=\left(\hat{H}_{k \ell}\right)_{k, \ell=0,1, \ldots}$ by the generating functions

$$
\begin{aligned}
& \sum_{\ell=0}^{\infty} \hat{H}_{k \ell}(2 \ell+1) z^{\ell} \\
& \quad=\frac{2^{n}}{n} 4^{(n-1) k} z^{-k-1}\left(1-\sqrt{1-4^{-n+1} z}\right)^{2 k+1}\left(1-4^{-n+1} z\right)^{-1 / 2},|z|<4^{n-1} .
\end{aligned}
$$

Then $\hat{H}$ is the unique upper triangular inverse of $\hat{G}$.

Proof. For every $\tau$ in the unit disc we have

$$
\sum_{r=0}^{\infty} \hat{H}_{k r}(2 r+1) 4^{r(n-1)} \tau^{r}=\frac{2^{-n+2}}{n} \frac{(1-\sqrt{1-\tau})^{k}}{(1+\sqrt{1-\tau})^{k+1}} \frac{1}{\sqrt{1-\tau}} .
$$

Now, for $t \in D_{n}$ and $\tau=T_{n}^{-2}(\beta(t))$, equation (3.48) becomes

$$
\sum_{r=0}^{\infty} \hat{H}_{k r} n(2 r+1) 2^{n(2 r+1)} t^{(2 r+1) n} \frac{1-t^{2 n}}{\left(1+t^{2 n}\right)^{2 r+2}}=2 t^{(2 k+1) n} .
$$

By equation (3.46), the above equation has the equivalent form

$$
\sum_{\ell=0}^{\infty} \sum_{r=0}^{\infty} \hat{H}_{k r} \hat{G}_{r m} t^{(2 m+1) n}=t^{(2 k+1) n},
$$

which implies that

$$
\sum_{r=0}^{\infty} \hat{H}_{k r} \hat{G}_{r m}=\delta_{k m}
$$

This lemma leads us to the next theorem whose proof is similar to that of Theorem 3.1 and therefore is omitted.

Theorem 3.2. Let $f \in A(R)$. Then for any $k \geq 0$

$$
A_{(2 k+1) n}(f)=\sum_{r=0}^{\infty} \hat{H}_{k r} \mathcal{L}_{2 r+1}(f) .
$$

Specializing (3.50) to the case $k=0$, we get

$$
A_{n}(f)=\sum_{r=1}^{\infty} \hat{H}_{0, r-1} \mathcal{L}_{2 r-1}(f) .
$$


Since

$$
\sum_{r=0}^{\infty} \hat{H}_{0 r}(2 r+1) z^{r}=\frac{2^{n}}{n} z^{-1}\left(\left(1-4^{-n+1} z\right)^{-1 / 2}-1\right)
$$

we conclude from (3.40) that

$$
\sum_{r=1}^{\infty} \hat{H}_{0, r-1}(2 r+1) z^{r}=\frac{2^{n+1}}{n} \sum_{r=1}^{\infty} r \alpha_{r} z^{r} .
$$

That is,

$$
\hat{H}_{0, r-1}=\frac{2^{n+1}}{n} \frac{r}{2 r-1} \alpha_{r}, \quad r \geq 1,
$$

and so equation (3.51) becomes

$$
A_{n}(f)=\frac{2^{n+1}}{n} \sum_{r=1}^{\infty} \frac{r}{2 r-1} \alpha_{r} \mathcal{L}_{2 r-1}(f) .
$$

In particular, this implies that

$$
A_{n}(f)=\frac{2^{n+1}}{n} \sum_{j=1}^{s} \frac{j}{2 j-1} \alpha_{j} \mathcal{L}_{2 j-1}(f)
$$

for $f \in \pi_{(2 s+1) n-1}$, a formula from [10, Theorem 4.2], where it was pointed out that (3.52) is of maximum degree of precision among all quadrature formulas of the type

$$
\sum_{k=0}^{2 s-1} \sum_{j=0}^{n} \lambda_{k j} f^{(k)}\left(x_{j, s}\right)
$$

4. Gauss-Turán quadrature formulas for weight functions in $\mathcal{W}_{n}$

In this section, we combine our observations of the two previous sections and derive Gauss-Turán quadrature formulas for any weight function $w \in \mathcal{W}_{n}$.

Our first result is

Theorem 4.1. Let

$$
\gamma_{j}=\sum_{\ell=0}^{j} H_{\ell j} \rho_{\ell}, \quad j=0,1,2, \ldots
$$

Then the Gauss-Turán quadrature of order $s$ for $w \in \mathcal{W}_{n}$ is given by

$$
I(f ; w)=\frac{\pi}{2} \sum_{j=0}^{s} \gamma_{j} \mathcal{L}_{2 j}(f), \quad f \in \pi_{2(s+1) n-1} .
$$

Proof. We eliminate the Fourier-Chebyshev coefficients from equations (2.5) and (3.37) to obtain the result.

As an addition to (2.9), we specialize (4.1) to the case $s=1$ and obtain the quadrature formula

$$
\int_{-1}^{1} f(x) w(x) d x=\frac{\pi \rho_{0}}{2 n} \sum_{j=1}^{n} f\left(\xi_{j}\right)+\frac{\pi\left(\rho_{0}+\rho_{1}\right)}{2 n 4^{n}} f^{\prime}\left(\xi_{1}^{2}, \ldots, \xi_{n}^{2}\right), f \in \pi_{4 n-1} .
$$


Here we used the fact that

$$
\left(\begin{array}{ll}
G_{00} & G_{01} \\
G_{10} & G_{11}
\end{array}\right)=\left(\begin{array}{cc}
\frac{n}{2} & -n \\
0 & n 4^{n}
\end{array}\right)
$$

so that

$$
\left(\begin{array}{ll}
H_{00} & H_{01} \\
H_{10} & H_{11}
\end{array}\right)=\left(\begin{array}{cc}
\frac{2}{n} & \frac{2}{n 4^{n}} \\
0 & \frac{1}{n 4^{n}}
\end{array}\right)
$$

It is easy to check that

$$
f^{\prime}\left(\xi_{1}^{2}, \ldots, \xi_{n}^{2}\right)=\frac{4^{n-1}}{n^{2}} \sum_{j=1}^{n}\left[\left(-\xi_{j}\right) f^{\prime}\left(\xi_{j}\right)+\left(1-\xi_{j}^{2}\right) f^{\prime \prime}\left(\xi_{j}\right)\right],
$$

and so, for any $f \in \pi_{4 n-1}$, we get from (4.2)

$$
\begin{aligned}
\int_{-1}^{1} f(x) w(x) d x= & \frac{\rho_{0}}{n} \sum_{j=1}^{n} f\left(\xi_{j}\right)-\frac{\left(\rho_{0}+\rho_{1}\right)}{4 n^{3}} \sum_{j=1}^{n} \xi_{j} f^{\prime}\left(\xi_{j}\right) \\
& +\frac{\left(\rho_{0}+\rho_{1}\right)}{4 n^{3}} \sum_{j=1}^{n}\left(1-\xi_{j}^{2}\right) f^{\prime \prime}\left(\xi_{j}\right) .
\end{aligned}
$$

We now provide a Gauss-Turán quadrature formula of highest degree of precision for $A_{n}(f)$.

Theorem 4.2. Let

$$
\mu_{\ell}= \begin{cases}\rho_{0}+\rho_{1}, & \ell=0 \\ \frac{1}{2}\left(\rho_{\ell+1}+\rho_{\ell}\right), & \ell \geq 1\end{cases}
$$

and

$$
\nu_{j}=\sum_{\ell=0}^{j} \hat{H}_{\ell j} \mu_{\ell}, \quad j \geq 0
$$

Then

$$
\int_{-1}^{1} f(x) T_{n}(x) w(x) d x=\frac{\pi}{2} \sum_{j=0}^{s} \nu_{j} \mathcal{L}_{2 j+1}(f), \quad f \in \pi_{(2 s+3) n-1} .
$$

Proof. First we recall that whenever

$$
f=\sum_{j=0}^{\infty} A_{j} T_{j}
$$

it follows that

$$
\begin{aligned}
f T_{n} & =\frac{1}{2} \sum_{j=0}^{\infty} A_{j}\left(T_{n+j}+T_{|n-j|}\right) \\
& =\frac{1}{2} \sum_{j=0}^{2 n} A_{j} T_{|n-j|}+\frac{1}{2} \sum_{j=n+1}^{\infty}\left(A_{j-n}+A_{j+n}\right) T_{j} .
\end{aligned}
$$

Hence we conclude that

$$
A_{2 \ell n}\left(f T_{n}\right)= \begin{cases}A_{n}, & \ell=0 \\ \frac{1}{2}\left(A_{(2 \ell-1) n}+A_{(2 \ell+1) n}\right), & \ell \geq 1\end{cases}
$$


Therefore, equation (2.5) implies that

$$
\begin{aligned}
\frac{2}{\pi} \int_{-1}^{1} f(x) T_{n}(x) w(x) d x & =\frac{\rho_{0}}{2} A_{n}+\frac{1}{2} \sum_{j=1}^{\infty} \rho_{\ell}\left(A_{(2 \ell-1) n}+A_{(2 \ell+1) n}\right) \\
& =\frac{\left(\rho_{0}+\rho_{1}\right)}{2} A_{n}+\sum_{\ell=1}^{\infty} \frac{1}{2}\left(\rho_{\ell+1}+\rho_{\ell}\right) A_{(2 \ell+1) n} \\
& =\sum_{\ell=0}^{\infty} \mu_{\ell} A_{(2 \ell+1) n} .
\end{aligned}
$$

We now use (3.50) to eliminate the Fourier-Chebyshev coefficients of $f$ to obtain

$$
\int_{-1}^{1} f(x) T_{n}(x) w(x) d x=\frac{\pi}{2} \sum_{j=0}^{\infty} \gamma_{j} \mathcal{L}_{(2 j+1)}(f),
$$

which is certainly valid when $f$ is a polynomial. Moreover, if $f \in \pi_{(2 s+3) n-1}$, then $\mathcal{L}_{2 j+1}(f)=0$ for $j>s$, whence (4.3) follows.

We conclude with some comments about the quadrature formulas studied here and also provide a convergence result for them.

Recall that the degree of exactness of any Gauss-Turán quadrature rule depends on the number $n$ and on the multiplicity $2 s+1$ of the nodes. Moreover, in general, the nodes vary both with $n$ and $s$. In contrast, the rules (4.1) have nodes independent of $s$. This allows one to get higher precision by increasing $s$, without recalculating the nodes. Obviously, when $s$ increases, more derivatives of $f$ are needed. However, in many cases, such evaluation can be performed using suitable relations between successive derivatives of the function under consideration [6].

A rather natural question arises at this point concerning the convergence of (4.1), for $s \rightarrow \infty$. With regard to this question, besides the general theorem in [10] another convergence result can be stated here.

To this end, we write the quadrature (4.1) in the form

$$
I(f ; w)=\frac{\pi}{2} \sum_{j=0}^{s} \gamma_{j} \mathcal{L}_{2 j}(f)+R_{s, n}(f ; w),
$$

where

$$
R_{s, n}(f ; w)=0 \quad \text { for } \quad f \in \pi_{2(s+1) n-1} .
$$

Theorem 4.3. Let $f \in C^{\infty}[-1,1]$ and put $\left|f^{(k)}(x)\right| \leq M_{k}, k \in N, x \in[-1,1]$; if

$$
\lim _{s \rightarrow \infty} M_{2(s+1) n} /\left(2^{(n-1)(2 s+1)}[2(s+1) n] !\right)=0,
$$

then

$$
\lim _{s \rightarrow \infty} R_{s, n}(f ; w)=0 .
$$

Proof. From (4.4) and the Peano theorem there exists $\tau \in(-1,1)$ such that

$$
\begin{gathered}
R_{s, n}(f ; w)=\frac{f^{(2(s+1) n)}(\tau)}{[2(s+1) n] !} \int_{-1}^{1} x^{n}\left[\prod_{i=1}^{n}\left(x-\xi_{i}\right)\right]^{2 s+1} w(x) d x, \\
\prod_{i=1}^{n}\left(x-\xi_{i}\right)=T_{n}(x) / 2^{n-1}
\end{gathered}
$$

equation (4.5) immediately follows. 


\section{REFERENCES}

1. V. Badkov, Convergence in the mean and almost everywhere of Fourier series in polynomials orthogonal on an interval, Math. U.S.S.R. - Sb. 24 (1974), 223-256. MR 50:7938

2. A. Ghizzetti and A. Ossicini, Quadrature Formulae, Academic Press, New York (1970). MR 42:4012

3. G.H. Golub and J. Kautsky, Calculation of Gauss quadrature with multiple free and fixed knots, Numer. Math. 41 (1983), 147-162. MR 84i:65030

4. L. Gori and M.L. Lo Cascio, A note on a class of Turán type quadrature formulas with generalized Gegenbauer weight functions, Studia Univ. Babeş - Bolyai Mathematica 37 (1992), 47-63. MR 95j:65020

5. L. Gori and E. Santi, On the convergence of Turán type rules for Cauchy principal value integrals, Calcolo 28 (1991), 21-35. MR 94e:65026

6. - On the evaluation of Hilbert transforms by means of a particular class of Turán quadrature rules, Numer. Algor. 10 (1995), 17-39.

7. O. Kis, Remarks on mechanical quadrature, (Russian), Acta Math. Acad. Sci. Hungaricae 8 (1957), 473-476. MR 20:196

8. C.A. Micchelli, The fundamental theorem of algebra for monosplines with multiplicities, in Linear Operators and Approximation, eds. P. Butzer and J.P. Kahane, B. Sz. Nagy, ISNM 20, Birkhäuser Verlag, 1971, pp. 372-379. MR 52:14758

9. C.A. Micchelli and T.J. Rivlin, Turán formulas and highest precision quadrature rules for Chebyshev coefficients, IBM Journal of Research and Development 16 (1972), 372-379. MR 48:12784

10. C.A. Micchelli and A. Sharma, On a problem of Turán : multiple node Gaussian quadrature, Rend. Mat. VII 3 (1983), 529-552. MR 86d:41032

11. G.V. Milovanović, Construction of s-orthogonal polynomials and Turán quadratures, in Approx. Theory III, Niš, 1987, (ed. G.V. Milovanović), Univ. Niš, 1988, pp. 311-328. MR 89g:65023

12. P. Nevai, Mean convergence of Lagrange interpolation III, Trans. A.M.S. 282 (1984), 669-698. MR 85c: 41009

13. P. Turán, On the theory of mechanical quadrature, Acta Sci. Math. Szeged 2 (1950), 30-37. MR 12:164b

14. 23-85. MR 82e:41003

Dipartimento di Metodi e Modelli Matematici, per le Scienze Applicate, Università "La Sapienza", Via Antonio Scarpa, 16-00161 Roma, Italia

Mathematical Sciences Department, iBM T.J. Watson Research Center, P.O. Box 218, Yorktown Heights, New York 10598 